\title{
Study of the Dependency of the Specific Power Absorption Rate on Several Characteristics of the Excitation Magnetic Signal when Irradiating a SPION-containing Ferrofluid
}

\author{
Alejandra Mina Rosales ${ }^{1 *}$, Elena Aznar ${ }^{2,3}$, Carmen Coll ${ }^{2}$, Rubén A. García Mendoza ${ }^{1}$, \\ A. Lorena Urbano Bojorge ${ }^{1}$, Nazario Félix González ${ }^{1}$, Ramón Martínez-Máñez ${ }^{2}$, \\ Francisco del Pozo Guerrero ${ }^{1,3}$, and José Javier Serrano Olmedo ${ }^{1,3}$ \\ ${ }^{1}$ Centre for Biomedical Technology (CTB), Universidad Politécnica de Madrid (UPM), Madrid, Spain. \\ Parque Científico y Tecnológico (UPM), Campus de Montegancedo, M40 km 38, 28223 Pozuelo de Alarcón, Madrid, Spain \\ ${ }^{2}$ Centro de Reconocimiento Molecular y Desarrollo Tecnológico (IDM). Unidad Mixta Universitat Politècnica de València - Universitat \\ de València, Spain, camí de vera $s / n, 46022$, Valencia, Spain \\ ${ }^{3}$ Biomedical Research Networking Center in Bioengineering Biomaterials and Nanomedicine (CIBER-BBN), Madrid, Spain
}

(Received 23 November 2015, Received in final form 25 August 2016, Accepted 25 August 2016)

\begin{abstract}
Magnetic hyperthermia mediated by superparamagnetic particles is mainly based in sinusoidal waveforms as excitation signals. Temperature changes are conventionally explained by rotation of the particles in the surrounding medium. This is a hypothesis quite questionable since habitual experimental setups only produce changes in the magnetic module, not in the field lines trajectories. Theoretical results were tested by changing the waveform of the exciting signal in order to compare non-sinusoidal signals against sinusoidal signals. Experiments were done at different frequencies: $200 \mathrm{KHz}, 400 \mathrm{KHz}, 600 \mathrm{KHz}, 800 \mathrm{KHz}$ and $1 \mathrm{MHz}$. Superparamagnetic Iron Oxide samples (SPION), made of magnetite $\left(\mathrm{Fe}_{3} \mathrm{O}_{4}\right)$ and suspended in water $(100 \mathrm{mg} / \mathrm{ml})$, were used. Magnetic field strength varies from $0.1 \pm 0.015 \mathrm{KA} / \mathrm{m}$ to $0.6 \pm 0.015 \mathrm{KA} / \mathrm{m}$. In this study was observed that the power loss depends on the applied frequency: for 1 to 2.5 RMS current the responses for each signal are part of the higher section of the exponential function, and for 3.5 to $8 \mathrm{RMS}$ current the response is clearly the decrement exponential function's tale (under $1 \times 10^{3} \mathrm{LER} / \mathrm{gr}$ ).
\end{abstract}

Keywords : magnetic hyperthermia, superparamagnetic iron oxide nanoparticle (SPION) ferrofluid, Specific Absorption Rate (SAR)

\section{Introduction}

Hyperthermia is a very commonly used technique in cancer treatment $[1,2]$ for its primary therapeutic effects and because it can help the release and even activation of some anticancer drugs. When cancer cells are heated at a temperature ranging from 42 to $45^{\circ} \mathrm{C}$, for a period of time of at least 30 minutes [3-7], they die due to the suffered hyperthermia. Magnetic Hyperthermia $(\mathrm{MH})$ is produced when an alternating magnetic field $(H)$ is applied on a ferrofluid which has been previously infused in the targeted tissue volume. In biomedical applications, these ferrofluids are made by suspending magnetic nanoparticles

(C)The Korean Magnetics Society. All rights reserved.

*Corresponding author: Tel: +34-913-36-46-45

Fax: +34-913-36-68-28, e-mail: alejandra.mina@ctb
(MNPs) in water. MNPs absorb energy from the alternating magnetic field and release part of it as heat. It is well-known that the thermic effect is enhanced when the ferrofluid is made with super-paramagnetic particles (SPIONs) with diameters between 10 and $50 \mathrm{~nm}[8,9]$. Due to the small dimensions of single-domain SPIONs (poor electrical conductivity), eddy currents can be neglected as a significant contribution in energy interchange. Therefore, the only phenomena involved in heat generation are two types of relaxation processes. Brownian relaxation produces heat by friction between SPIONs and a viscous fluid; particles are free enough to be able to rotate following the main alternating magnetic field. The other process involves rotation of the magnetic moment of each particle following the applied magnetic field and is known as Néel relaxation $[3,8,10,11]$. Brownian relaxation is characterized by the parameter $\tau_{B}(\mathrm{~s})$ whilst Néel relaxation 
is characterized by $\tau_{N}(\mathrm{~s})$. When particles are suspended in water, both processes are combined to produce the net relaxation time $\left(\tau_{R}\right)$ as follows:

$$
\frac{1}{\tau_{R}}=\frac{1}{\tau_{B}}+\frac{1}{\tau_{N}}
$$

In the case of superparamagnetic particles, $\tau_{R}$ is determined by $\tau_{N}$. $[12,13,14]$. Under the assumption of a rotating magnetic field, the power loss can been calculated as

$$
P=\pi \mu_{0} \chi_{0} H_{0}^{2} f \frac{2 \pi f \tau_{R}}{1+\left(2 \pi f \tau_{R}\right)^{2}}
$$

Where $\mu_{0}$ is the free space permeability, $\chi_{0}$ is the equilibrium susceptibility of the particles, $H_{0}$ is the amplitude of the magnetic field, and $f$ is the rotation frequency.

In common experimental setups, the field is produced by a coil of several turns with a current of some amperes following a sinusoidal waveform. Therefore, the particles are immerse in a magnetic field that changes its direction (up and down) and magnitude. Continuous rotation, transient movements or changes on the position of the particle in the medium, can take place due to Brownian relaxation mechanism. These phenomena could be seen as a fixed particle in a randomly changing field, what has nothing to do with the established concept of rotating fields, which produces a stationary rotation of the particle in the medium. The theoretical concept of this steady state are predicted and explained by Brownian and Néel relaxations processes; however, there are transient rotations which are different from this theoretical concept. While stationary rotations take place when the excitation signal follows a sinusoidal waveform, transient rotations occur when the signal has a square shape.

In this work, the efficacy of hyperthermia was tested using several non sinusoidal signals. The effectiveness of the experiments was measured using the real power loss, equation (2). Results were compared against, well studied, sinusoidal waveform. Different frequencies and current intensities were tried to obtain the parameters that produce the maximum thermal effect using the same sample as target tissue.

\section{Materials and Methods}

The equipment involved in the present work is part of the Functional Characterization of Magnetic Nanoparticles Platform, a facility of the Biomedical Networking Center for Bioengineering, Biomaterials and Nanomedicine (CIBER-BBN) of the Institute of Health Carlos III in Madrid, Spain. The hyperthermia equipment is a home- made hyperthermia device that includes a calorimeter thermally characterized. It have been measured the power losses from experiments changing the frequency of the alternating magnetic field, the signal waveform and the value of the maximum field intensity.

\subsection{Material synthesis and characterization}

Superparamagnetic iron oxide nanoparticles (SPION) were synthesized following the method described by Lin and coworkers. Griri S [15]. In this procedure, a mixture of iron (II) chloride tetrahydrate $\left(498.0 \mathrm{mg}, 2.50 \times 10^{-3}\right.$ mol) and iron (III) chloride hexahydrate (810.0 mg, 22.99 $\times 10^{-3} \mathrm{~mol}$ ) were added to a deoxygenated aqueous solution $(600.0 \mathrm{~mL}, \mathrm{pH} 1.5)$. Then, $\mathrm{pH}$ of the solution was increased until 9 by adding drop wise an ammonium hydroxide aqueous solution $(1.50 \mathrm{M})$ under vigorous stirring. The resulting black precipitate was separated by centrifugation, washed with water until neutral $\mathrm{pH}$, and with ethanol. Finally, the resulting solid was dried to get a $\mathrm{Fe}_{3} \mathrm{O}_{4}$ powder. Synthesized SPION were characterized by TEM and powder X-ray diffraction. Representative micrograph of the solid shows nanoparticles with an average

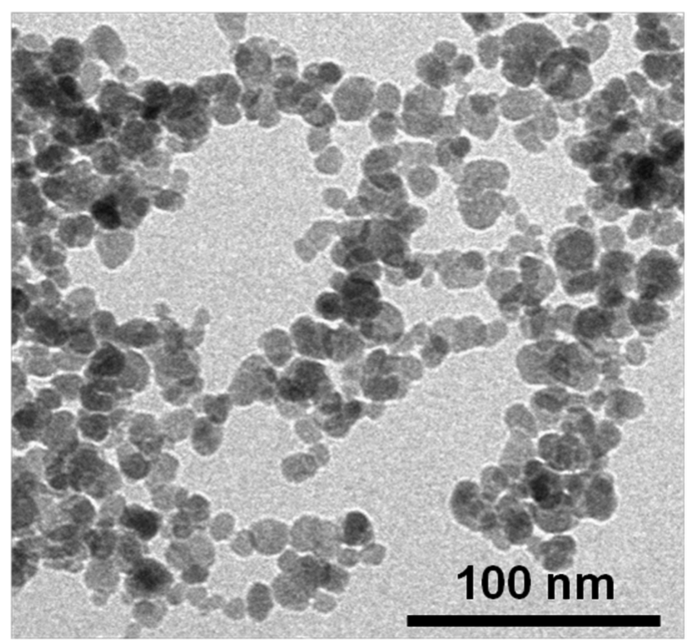

Fig. 1. TEM representative image of synthesized SPION Powder X-ray diffraction pattern.

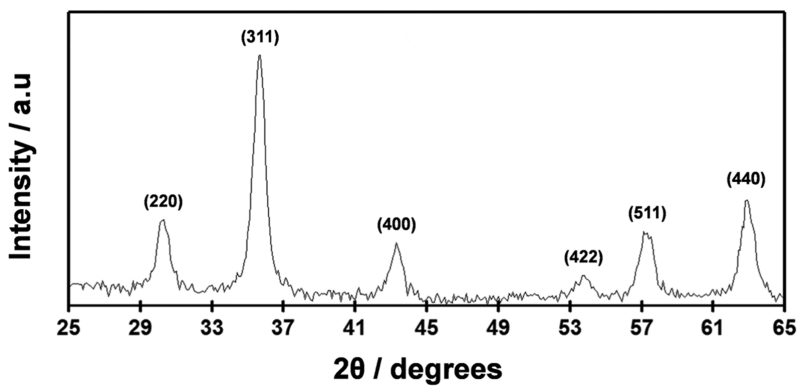

Fig. 2. Powder X-ray diffraction pattern. 


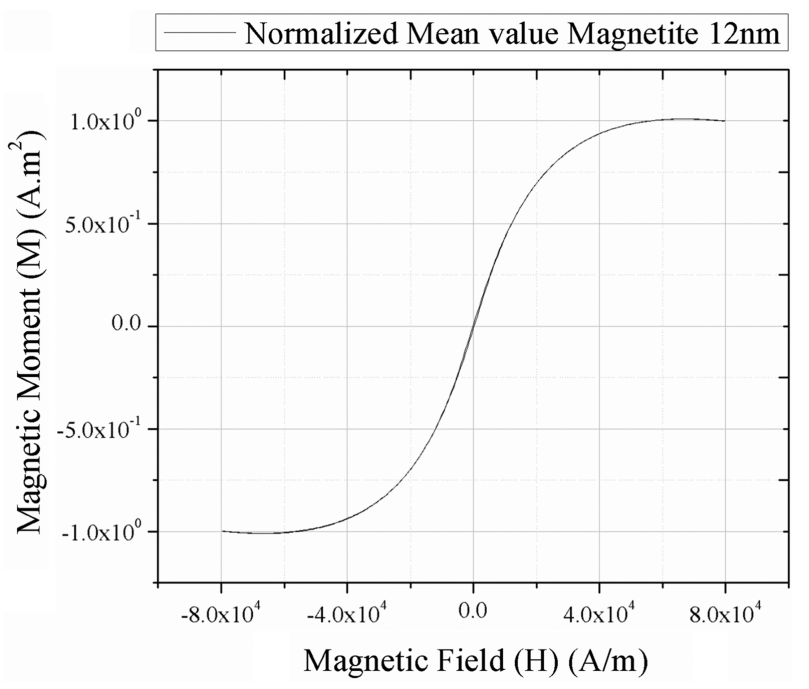

Fig. 3. $\mathrm{M}-\mathrm{H}$ curve of magnetite $\left(\mathrm{Fe}_{3} \mathrm{O}_{4}\right)$.

diameter of $14 \mathrm{~nm}$. Powder XRD pattern shows the typical reflections of iron oxide in the range $25^{\circ}<2 \theta<$ $65^{\circ}$. Thus, the corresponding Bragg peaks can be indexed with a cubic array of magnetite.

\subsection{Magnetic characterization of the sample}

The magnetic behavior of the MNPs from magnetite $\left(\mathrm{Fe}_{3} \mathrm{O}_{4}\right)$ was characterized by an Alternating Gradient Magnetometer (MicroMagTM 2900 AGFM System). In order to know the magnetic response, magnetic measurements (M-H curves) were performed to obtain the sample's magnetic moment through the measurement of the hysteresis loops. The measurements were taken at room temperature and a magnetic field strength of $80 \mathrm{kA} / \mathrm{m}$ was applied.

Figure 3 shows the magnetic moment of the magnetite. It can be observed a superparamagnetic behavior in the sample.

The magnetic characterization of the MNPs has been performed in the ICTS "NANBIOSIS", more specifically by the Unit U15 (Functional Characterization of Magnetic NPs Unit) of the CIBER in Bioengineering, Biomaterials \& Nanomedicne (CIBER-BBN) at the Technical University of Madrid.

\subsection{Hyperthermia Device Specifications}

The equipment used in this work is a homemade device. Figure 4, shows the experimental setup. A function generator (Tektronix AFG3022B) controlled by LabVIEW software was used to design and select the excitation signal. The signal goes through a power amplifier (1140 LA electronics \& innovation Ltd.) that works with frequencies ranging from $9 \mathrm{kHz}$ to $2 \mathrm{MHz}$, with output

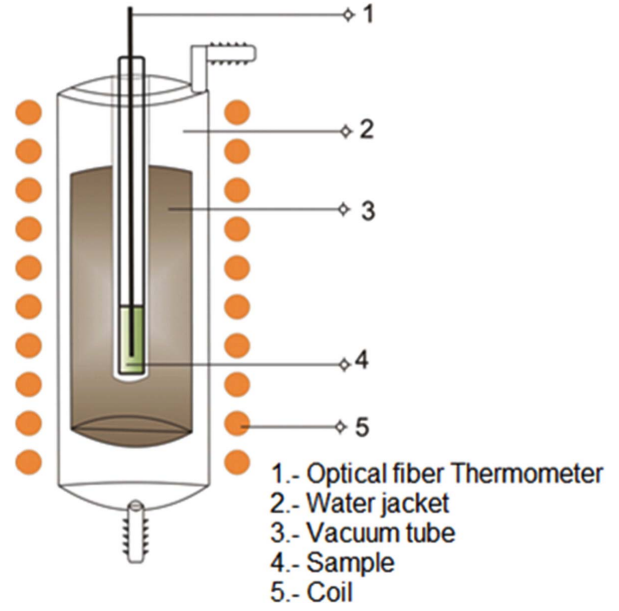

Fig. 4. (Color online) Diagram of the system where the magnetic ferrofluid was placed, height $13 \mathrm{~cm}$, radius of the outer tube $2.5 \mathrm{~cm}$ (water jacket), the middle tube ratio $1.5 \mathrm{~cm}$ (vacuum tube), radius of the inner tube $0.575 \mathrm{~cm}$ (sample).

saturation above $1000 \mathrm{~W}$ in the range of $9 \mathrm{kHz}$ to 100 $\mathrm{kHz}$; and above $1500 \mathrm{~W}$ in the range of $100 \mathrm{kHz}$ to 2 $\mathrm{MHz}$. The load is a copper coil ( $\varnothing 5 \mathrm{~mm}, 10$ turns) in which axis the calorimeter is placed with the sample inside. It was required to install some real load to compensate the pure reactive load of the coil, especially in the lower frequency range.

The probe is a calorimeter made from borosilicate glass which provides an adiabatic environment: an outer volume made full of running water, a vacuum volume in the middle, and an inner volume for the sample. The temperature of the water is regulated with the help of a thermostatic bath (DIGIT-COOL 3001373). The inner volume is blocked with a plug in order to reduce the heat interchange with the exterior. A temperature sensor runs through another thinner tube form outside to the center of the sample volume of ferrofluid, where the temperature is sensed.

The current in the coil is measured by means of a two turns coil around the main cable producing the magnetic field. The magnetic field inside the coil is calculated from the geometry of the coil and the measured current. A digital Oscilloscope (Tektronix, TDS 2024B) was used to measure all the electrical signals. Finally, the temperature is registered using an optical temperature sensor with an uncertainty of $0.02^{\circ} \mathrm{C}$ (Luma Sense Technologies m3300, Luxtron).

\subsection{Calorimeter calibration}

To characterize the calorimeter, a thermal model was used. Its formulation consists of three parameters: a power source $\left(P_{W}\right)$, a thermal conductance $\left(C_{D}\right)$ and a thermal 
capacitance $\left(C_{T}\right)$. Under the action of a continuous magnetic induction over ferrofluids, the power source is constant in time. Then, the increase of temperature $\Delta T(t)$ grows in time, $t$, following the function:

$$
\Delta T(t)=\Delta T_{\infty} \cdot\left[1-e^{\left(-t / \tau_{T}\right)}\right]
$$

Where the resulting temperature change $\Delta T_{\infty}$ in steady state can be related to the power source in two ways:

$$
\Delta T_{\infty}=P_{w} / C_{D}
$$

in the steady state, and

$$
\Delta T_{\infty}=\left.\frac{d(\Delta T(t))}{d t}\right|_{t=0} \cdot \tau_{T}
$$

where $d(\Delta T(t)) /\left.d t\right|_{t=0}$ is de slope of the rising temperature curve $\Delta T(t)$ at the beginning of the magnetic induction and $\tau_{T}$ is the characteristic time of the curve, so that

$$
C_{T}=\tau_{T} \cdot C_{D}
$$

The derivative method to calculate powers is only useful when the capacitance is extremely high, virtually infinite. Otherwise, the noise and the slight, but real, bending of the curve at the beginning could lead to miscalculations of the slope. In this experimental setup, the method of the steady state was used; even though the experiments are very long to calculate powers. However, this method has another risk: the parameter $C_{D}$ is also quite variable. Very small misplacing of the temperature probe, of some tenths of a millimetre, drives to very different measurements of these parameters. Contrarily, the capacitance and the characteristic time are quite easy to measure. Therefore, It has been used a combine approximation to the calculation of the power using the formula:

$$
\Delta T_{\infty}=\tau_{T} \cdot P_{w} / C_{T}
$$

Others parameters were necessary for to calculate the power, which is described then.

Deionized water-sample of $250 \mu \mathrm{l}$ were used and the sheltering water flux was fixed to $20^{\circ} \mathrm{C}$ to set the background temperatures for $\Delta T(t)$. It has been used a small surface mounting resistor as power source to heat the sample. The capacitance strongly depends on the material being heated, although the conductance is much more dependent on the boundaries. So, It has been assumed in our experimental setup that the resistor would affect much higher to the capacitance parameter that to the conductance one. First $\Delta T_{\infty}$ was taken and recorded for the given resistor power $P_{w}$. After removing the resistor, the characteristic time $\tau$ has been measured as the while the sample cools down. The cooling downs follows the

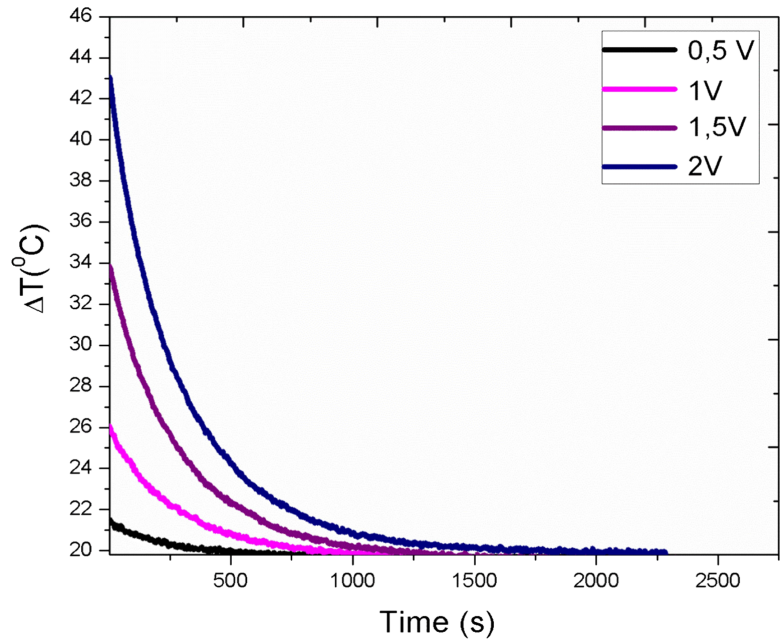

Fig. 5. (Color online) Cooling down for different after heating with different voltajes applied to the resistor.

formula (8) and in Fig. 5 can be experimentally seen four cooling down responses for different voltages:

$$
\Delta T(t)=\Delta T_{\infty} \cdot e^{\left(-t / \tau_{T}\right)}
$$

It has been checked the difference between the characteristic times from heating up to cooling down.

It has been applied different voltages to different resistor (of different sizes and nominal values) to obtain the parameters we needed. They result to be: $t=299.07 \pm$ $3.28(s), C_{D}$ de $0.0060 \pm 2.7 \mathrm{E}-4 \mathrm{~W} / \mathrm{K}, C_{T}=1.7948 .856 \mathrm{E}-$ $4 \mathrm{~J} / \mathrm{K}$. Different samples of water with different volumes produce different values. This is negligible in our study because ferrofluids have the same volume. Changes in capacitance and conductance due to the dispersion of nanoparticles in the water volume would change the parameters, especially the capacitance, yielding new values.

\subsection{Heating experiments with ferrofluids}

In all the experiments the ferrofluid volume was $250 \mu 1$, at a concentration of $100 \mathrm{mg} / \mathrm{mL}$ NPs suspended in water. The thermal isolation barrier was filled with a constant flow of water at $20^{\circ} \mathrm{C}$. The frequencies applied were 200 $\mathrm{kHz}, 400 \mathrm{kHz}, 600 \mathrm{kHz}, 800 \mathrm{kHz}, 1 \mathrm{MHz}$. It was defined different signal waveform, that were called signals $G, K$, $L, J$, as shown in Fig. 6(a), (c), (e), and (g) depicts the ideal entry signal, and (b), (d), (f), (h), the signal that came out from the amplifier to the coil. The sinusoidal signal is the reference for the others. Other signals were chosen to produce many slopes, as high as possible, plus their distortion as can be seen in Fig. 6 items (b), (d), (f) and (h). The magnetic field was uninterruptedly applied during 25 minutes, time enough to produce an observable steady state in $\Delta T$, and the temperature rising registered. 

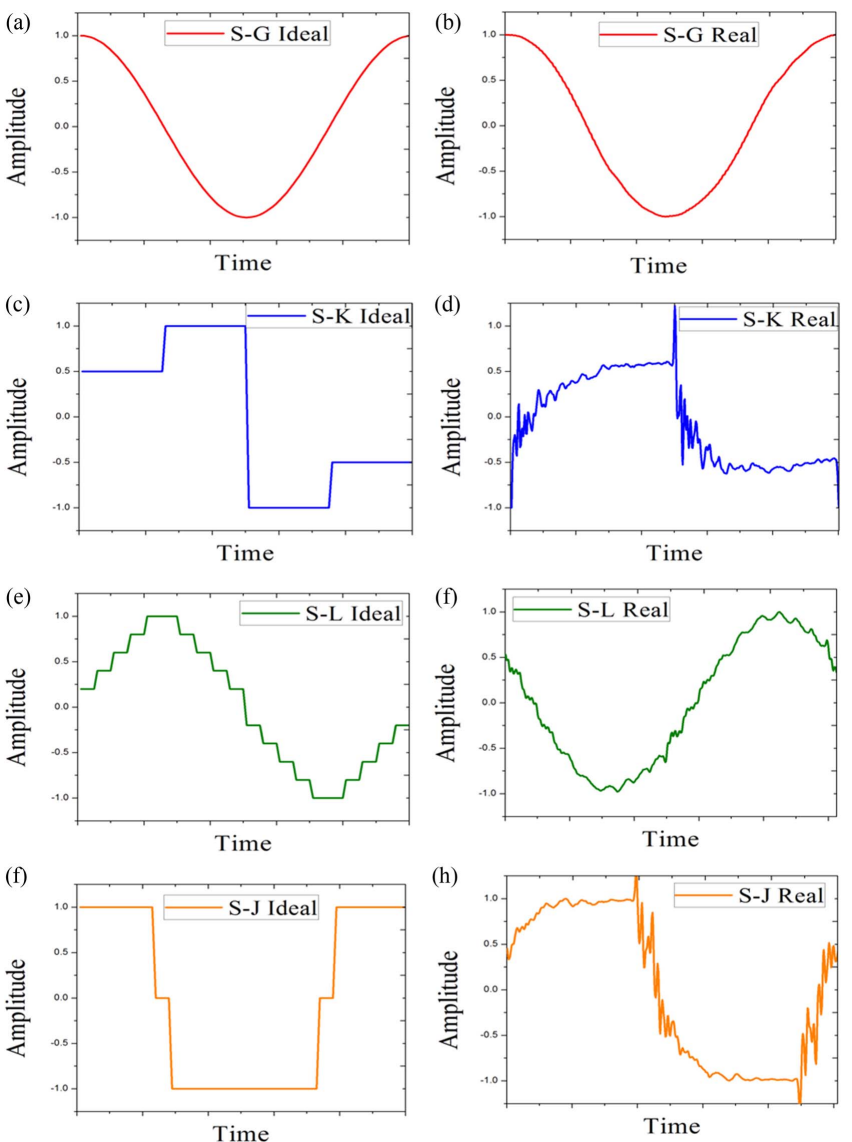

Fig. 6. (Color online) (a) Signal G ideal, (b) Signal G real, (c) Signal K ideal, (d) Signal K real, (e) Signal L ideal, (f) Signal $\mathrm{L}$ real, (g) Signal J ideal, (h) Signal J real.

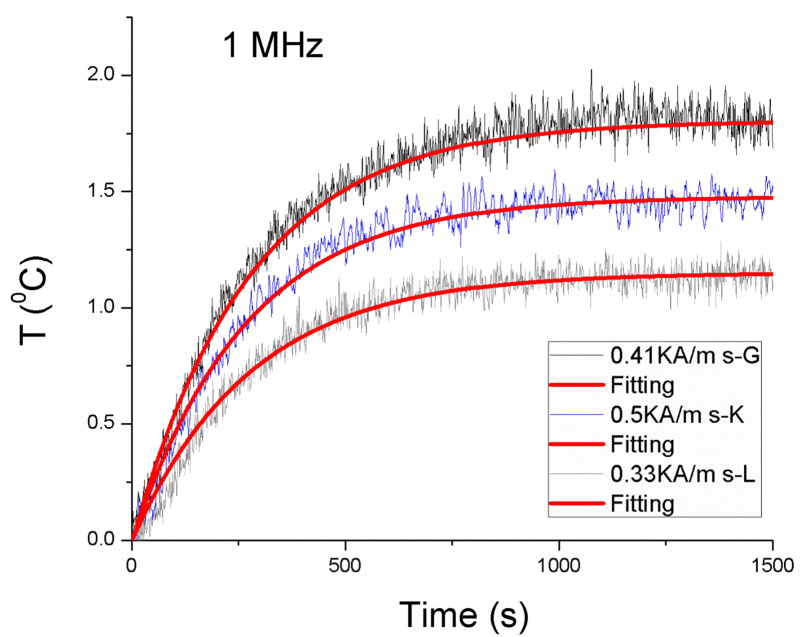

Fig. 7. (Color online) Examples of temperature measurements behavior and fittings with formula 3 .

Afterwards, the magnetic field was stopped and the cooling down registered too.

The raw measurements were of the type seen in Fig. 7.
Using the fittings and our previous calibration, it is possible to calculate the ferrofluid power, $P_{W, F F}$, heating the sample as

$$
P_{W, F F}=\Delta T_{\infty, F F} \cdot C_{T} / \tau_{F F}
$$

where $\Delta T_{\infty, F F}$ is the steady state increment in temperature and $\tau_{F F}$, the characteristic time of the rising curve, both obtained from the fitting. Our power are probably a bit underestimated since $C_{T}$, for the experimental set with only pure water, obtained from the calibration, should be a bit smaller than the real $C_{T}$, than using ferrofluid instead of water, because of the substitution of part of the water by iron oxide as compared to the calibration procedure.

\section{Results}

Specific Absorption Rate (SAR) ( $\mathrm{mW} / \mathrm{mg}$ ) is mathematically defined, $S A R=C_{T} d T /\left.d t\right|_{t=0}$ where $d T /\left.d t\right|_{t=0}$ is the temperature increase per unit of time at the beginning of the heating. In [6] and [16] is the more common method for measurement the rise of temperature.

However, it has been defined and hereafter used a derived figure of merit, the so called Loss Equivalent Resistance (LER):

$$
L E R=\frac{P_{w, F F}}{I^{2}}
$$

where $I$ is the root mean square current in the coil and $P_{w, F F}$ is the power heating the ferrofluid. The magnetization at so low magnetic fields hardly reaches the saturation. Since the excitation depends on the current amplitude, it is reasonable to normalize the heating power by the square of the current. Actually it is better to use the RMS current value for the shake of comparison between different exciting signals. Formula (2) already takes this kind of current, therefore by removing the current in the coil; one removes a variable in principle only depending on the experimental conditions. Different experiments using different currents should scale as those currents, but become comparable with our formalism.

This figure can be normalized again to remove the extensive effect of the amount of iron oxide by eliminating its weight as follows:

$$
L E R / g r=\frac{P_{w, F F}}{\text { weight } \times I^{2}}\left[\frac{W}{g r \times A^{2}}\right]
$$

It is easy to see then, that the commonly used SAR is and our LER/gr relates each other as follows:

$$
L E R / g r=S A R / I_{R M S}^{2}
$$

The obtained values are represented in Fig. 8 for 1 


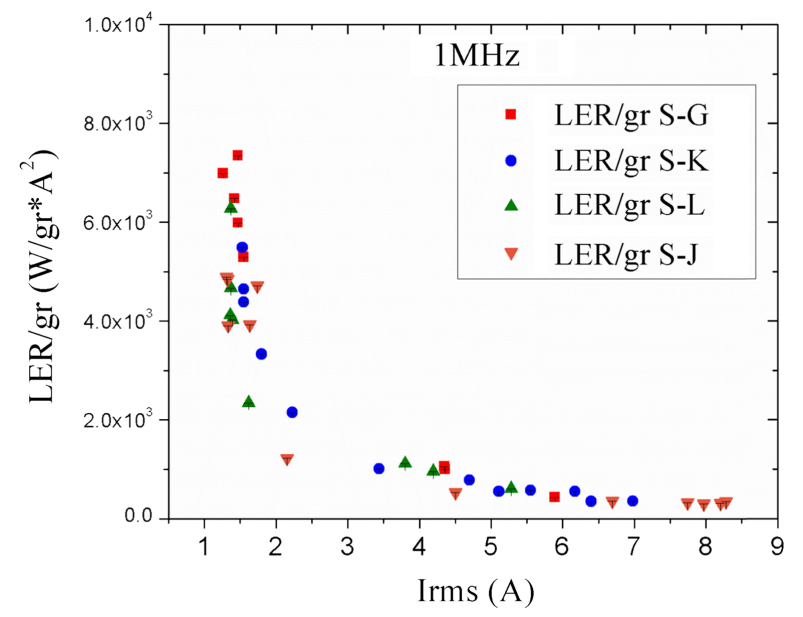

Fig. 8. (Color online) LER/gr in function of the RMS current in the coil at $1 \mathrm{MHz}$ for different signals showing: double linear plot.

MHz. There are two distinguishable areas, low (1-2.5 RMS current) and high (3.5-8 RMS currents). It seems that the LER limits for high current. However, this is not true as can be seen in the Figs. 9(a)-(e).

In Figs. 9(a) to 9(e) have been showed the fittings for the LER on the current as following, from pure obser-

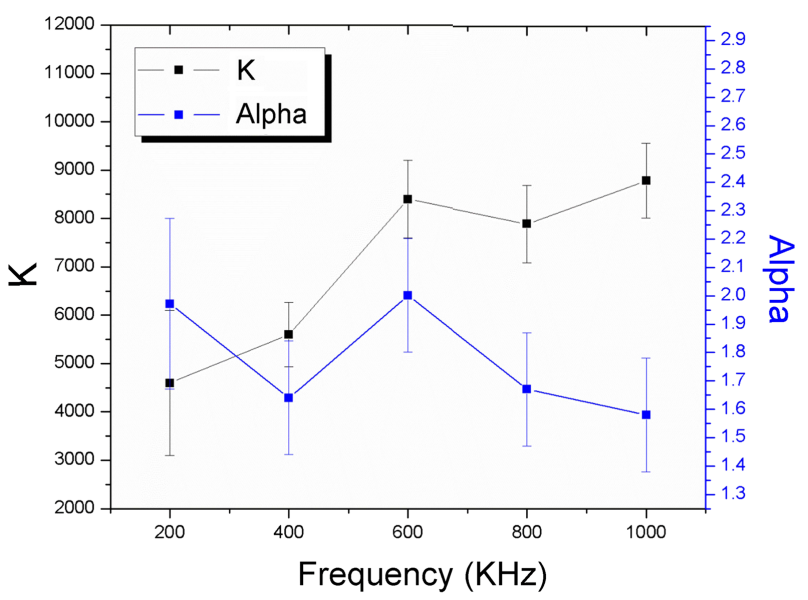

Fig. 10. (Color online) $K$ and $\alpha$ in function of the all the frequencies (phenomenological fitting, the unit was discarded).

vation, the trend: $L E R / g r=K /(\operatorname{Irms})^{\alpha}$. $K$ is approximately the invariable with the frequency. However, the exponent clearly grows with lowering frequencies from 1.58 up to 2.13. In the case of lower frequencies, our equipment works worse giving rise to higher noise in our measurements.

The device's behavior about applied current is directly
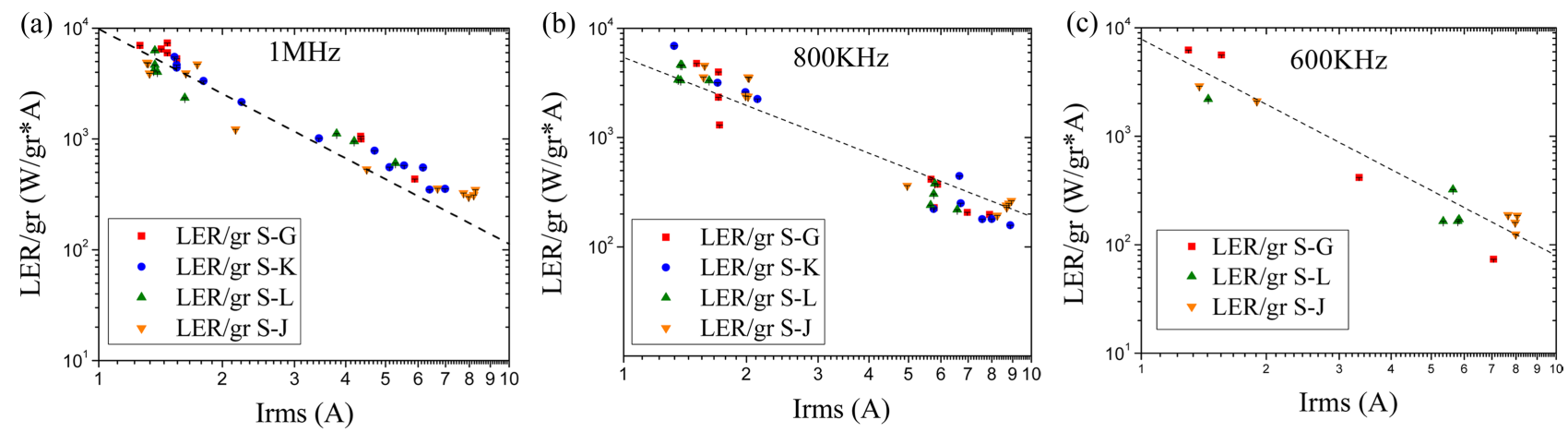
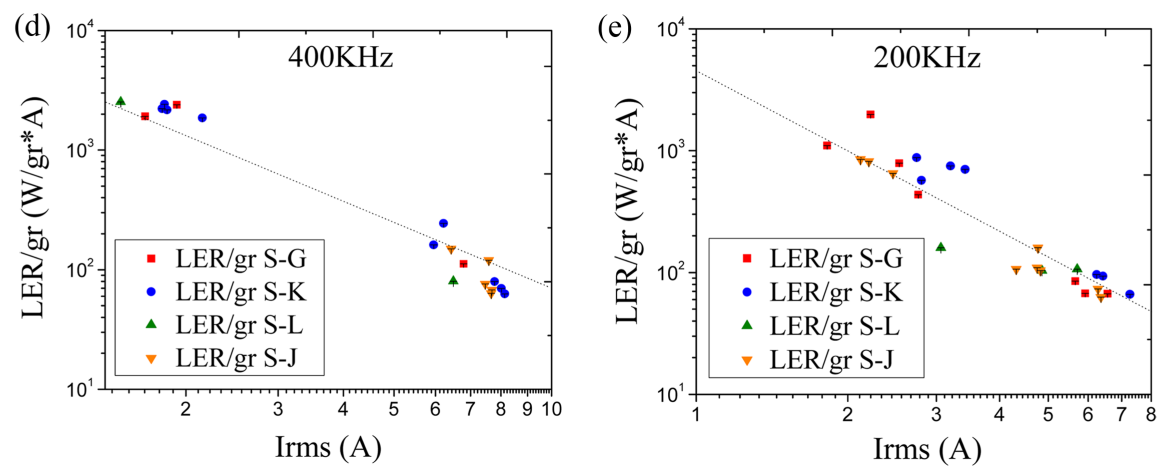

Fig. 9. (Color online) (a): LER/gr in function of the RMS current in the coil at $1 \mathrm{MHz}$ for different signals: $\mathrm{K}=8.8 \pm 0.8 \times 10^{3}$, $\alpha=1.6 \pm 0.2$, (b) $\mathrm{LER} / \mathrm{gr}$ in function of the RMS current in the coil at $800 \mathrm{kHz}$ for different signals: $\mathrm{K}=7.9 \pm 0.8 \times 10^{3}, \alpha=$ $1.7 \pm 0.2$. (c) $\mathrm{LER} / \mathrm{gr}$ in function of the RMS current in the coil at $600 \mathrm{kHz}$ for different signals: $\mathrm{K}=8.7 \pm 2.5 \times 10^{3}, \alpha=2.1 \pm 0.7$. (d) LER/gr in function of the RMS current in the coil at $400 \mathrm{kHz}$ for different signals: $\mathrm{K}=5.6 \pm 0.7 \times 10^{3}, \alpha=1.6 \pm 0.2$. (e) LER/gr in function of the RMS current in the coil at $200 \mathrm{kHz}$ for different signals: $\mathrm{K}=4.6 \pm 1.5 \times 10^{3}, \alpha=2.0 \pm 0.3$. 


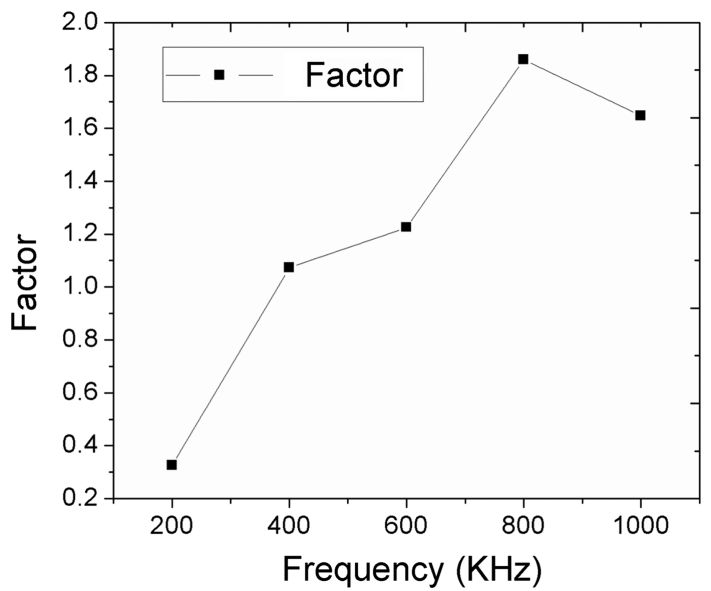

Fig. 11. LER/gr for currents of $8 \mathrm{~A}$ in function of the frequency can be given as a FACTOR times the theoretical value given by the theoretical approach (2) using a relaxation parameter $\tau$ of $5.5 \mathrm{e}^{-9} \mathrm{~s}$.

proportional to the signal distortion, the first assumption was that the flanks of the altered signal would produce greater alignment of the axes of anisotropy respecting to magnetic field and therefore more temperature, however the results showed that these flanks due to the distortion was negligible for magnetic moment of the particle.

The final calculus is considering the comparison of the theoretical results with those obtained in the experiment, and taking formula (2), where only the variable parameters $f$ and $\tau_{R}$ are considered, the equation remains as $\frac{2 \pi f^{2} \tau_{R}}{1+\left(2 \pi f \tau_{R}\right)^{2}}$, this value is divided by 8 Amp (RMS current) and the theoretical LER is obtained and shown in Fig. 11, has been gotten by dividing $\mathrm{LER}_{\text {Theoretical }}$ and $\mathrm{LER}_{\text {Experiment }}$ for near current taking $8 \mathrm{Amp}$ on each frequency. However, despite the noise, which can lead to not so trustable results in exact figures, the trend to reduce the LER value for increasing currents is clear, at least until certain values that this equipment could achieve. Actually, if we put attention to higher values, for about 8-9 A, the LEP looks like reaching a stable value, at least for higher frequencies. These values are shown in Fig. 11.

\section{Discussion}

There are two clear trends arising from our data that we do observe. The first one is that the power loss is not independent from the frequency, but it has a trend to grow for growing frequencies.

The second trend is the dependence of the normalized power loss on amplitude of the magnetic field: it is higher for very low currents (1-2.5 RMS Current) than for higher currents (3.5-8 RMS Current). At large currents (magnetic fields) the normalized power loss behaves as expected: it tends to be independent from the applied current, but it grows for decreasing currents. The fitting formula is only a phenomenological one, arbitrarily chosen to provide a good fit without theoretical support.

For high enough fields $(15 \mathrm{kA} / \mathrm{m}$ maximum for medical applications), especially at higher frequencies, the particles are aligned with the exciting field almost all the time along the excitation cycle. That is, there is some-like threshold magnetic field intensity above which the particle magnetic moment and the particle easy axis are both parallel to the external field. No losses can be produced along the time lasting that situation. Only part of the cycle is, then, useful to heat the sample. However, between the threshold band extremes (the field changes its direction along the excitation time cycle, so the threshold acts twice along the same cycle) the particles continuously move because the field strength is not enough $(0.6 \pm 0.015 \mathrm{KA} /$ $\mathrm{m}$ maximum for this study) to stop them parallel to the field lines, then taking place the characteristic frictions that lead to the losses, which finally heat the sample. For higher fields, only part of the cycle is active to produce heat, whilst for lower fields, the whole cycle is useful. Moreover, the lower the field, the less aligned are the particles, so they are able to produce more heat per unit of magnetic field magnitude.

\section{Conclusions}

According to this idea, different waveforms should produce different power losses since the phenomenon would be more a transient one than a steady-state effect. High exiting signal slopes could induce higher power losses, without needing to use very high magnetic field peaks. On the contrary, peaks no higher than the threshold would be necessary to optimize the power loss, whereas the slope is abrupt enough. Finally, for low currents ( 1 a 2.5 RMS) have been recovered the independence of the power loss forms the coil current. It has not been reached this situation because the temperature increases where below our experimental resolution. This is the new hypothesis that deserves to be contrasted in future works.

Several trends have been found what deserve more detailed attention, in what we are working now by doing a new experimental set. The dependence of the power loss on the frequency for certain nanoparticles in a given media could serve to measure the characteristic relaxation time. Similarly the hypothesis about the threshold magnetic field and its possible relation to the excitation field waveform open the door to more efficient ways to pro- 
duce magnetic hyperthermia.

\section{References}

[1] Z. Wu, Z. Zhuo, D. Cai, J. A. Wu, J. Wang, and J. Tang, Tech. and Health Care 23, 203 (2015).

[2] C. A. Quinto, P. Mohindra, S. Tong, and G. Bao, Nanoscale 7, 12728 (2015).

[3] S. Mornet, S. Vasseur, F. Grasset, and E. Duguet, J. Mater. Chem. 14, 2161 (2004).

[4] R. Hergt, W. Andrä, C. G. d'Ambly, I. Hilger, and W. A. Kaiser, U. IEEE Trans. Magn. 34, 3745 (1998).

[5] P. Pradhan, J. Giri, G. Samanta, H. D. Sarma, K. P. Mishra, J. Bellare, R. Banerjee, and D. Bahadur, J. Biomed. Mater. Res. 81B, 12 (2007).

[6] A. Jordan, R. Scholz, P. Wust, H. Fahling, and R. Felix, J. Magn. Magn. Mater. 201, 413 (1999).
[7] Q. A. Pankhurst, J. Conolly, S. K. Jones, and J. Dobson, J. Phys. Appl. Phys. 36, R167 (2003).

[8] X. Wang, H. Gu, and Z. Yang, J. Magn. Magn. Mater. 293, 334 (2005).

[9] S. Bedanta and W. Kleemann, J. Physics, 42 (2009).

[10] V. S. Kalambur, B. Han, B. E. Hammer, T. W. Shield, and J. C. Bischof, Nanotechnology 16, 1221 (2005).

[11] K. Hu, J. Sun, Z. Guo, P. Wang, Q. Chen, M. Ma, N. Gu, Advan. Mater. 27, 2507 (2015).

[12] H. Mamiya, J. Nanomaterials 2013, (2013).

[13] R. E. Rosensweig, J. Magn. Magn. Mater. 252, 370 (2002).

[14] I. M. Obaidat, B. Issa, and Y. Haik, Nanomaterials 5, 63 (2015).

[15] S. Giri, B. G. Trewyn, M. Stellmaker, and V. Lin, Angew. Chem. Int. Ed. 44, 5038 (2005).

[16] F. Shubitidze, K. Kekalo, R. Stigliano, and I. Baker, J. Appl. Phys. 117, 094302 (2015). 